\section{Análise da sobrecarga de cuidadores de jovens com síndrome de down fisicamente ativos e sedentários}

\section{Overload analysis of caregivers of youths with down syndrome physically active and sedentary}

Carolina Ventura Fernandes Pasetto

Bruna Barboza Seron ${ }^{1}$

Everaldo Lambert Modesto'

Eloise Werle de Almeida'

Elaine Cappellazzo Souto²

Márcia Greguol’

\section{RESUMO}

A sobrecarga de cuidadores de pessoas com deficiência tem sido foco de atenção de pesquisadores preocupados com a saúde e qualidade de vida desta população. O objetivo deste estudo foi avaliar a sobrecarga de cuidadores de jovens com Síndrome de Down (SD) e a possível influência de dois programas de exercícios físicos sobre esta variável. Participaram do estudo 38 adolescentes com SD e seus cuidadores. Entre os jovens, 15 foram submetidos a um programa de treinamento aeróbio, 14 a um programa de treinamento resistido e 9 fizeram parte do grupo controle. Os cuidadores responderam ao Burden Interview para a percepção da sobrecarga antes e após o período de intervenção de 12 semanas. Os dados foram comparados por meio de teste $t$ pareado e ANOVA, com significância $p \leq 0,05$. A sobrecarga manifestada pelos cuidadores foi moderada e não foram vistas diferenças significantes nos momentos pré e pós-intervenção para nenhum dos grupos, nem tampouco diferenças entre os grupos. Foi verificada uma associação significativa entre a sobrecarga e o nível socioeconômico, mostrando que cuidadores de classes mais baixas percebem maior sobrecarga. Embora o exercício físico não tenha sido suficiente para impactar a sobrecarga dos cuidadores, esta prática deve ser incentivada para jovens com síndrome de Down.

\section{PALAVRAS-CHAVE}

Sobrecarga; Cuidadores; Síndrome de Down; Exercício físico.

\begin{abstract}
The overload of caregivers of people with disabilities has been the focus of researchers concerned with the bealth and quality of lives of this population. The aim of this study was to assess the overload of caregivers of youths with Down Syndrome (DS) and the possible influence of two physical exercise programs on this variable. The study included 38 adolescents with DS and their caregivers. Among youths, 15 were subjected to an aerobic training program, 14 to a resistance training program and 9 took part of the control group. Caregivers responded to Burden Interview to the perception of overload before and after an intervention period of 12 weeks. Data were compared by paired $t$-test and ANOVA with $p<0.05$. The overload expressed by caregivers was moderate and no significant difference was detected between pre and post intervention evaluations for any of the groups, nor between groups. A significant association between overload and socioeconomic status were observed, showing that caregivers of lower classes perceived higher overload. Although exercise has not been enough to impact on caregiver burden, this practice should be encouraged for young people with Down syndrome.
\end{abstract}

\section{KEYWORDS}

Overload; Caregivers; Down Syndrome; Physical exercise.
Rev Bras Ativ Fis Saúde p. 636-646 DOI

http://dx.doi.org/10.12820/rbafs.v.19n5p636

1 Universidade Estadual de Londrina, Centro de Educação Física e Esporte, Londrina, Paraná, Brasil

2 Universidade Federal da Paraíba, Departamento de Educação Física, João Pessoa, Paraíba, Brasil 


\section{INTRODUCCÃO}

O termo "sobrecarga familiar/cuidadores" foi introduzido para descrever as consequências adversas de cuidar de pacientes com distúrbios psiquiátricos severos e que foi sucessivamente exportado para o âmbito da deficiência intelectual (DI) ${ }^{1}$. Recentemente, alguns autores propõem o termo "impacto" como alternativa ao termo "sobrecarga", por verificarem que nem sempre para a família o ato de cuidar é problemático, podendo, ao contrário, apresentar também consequências positivas ${ }^{1,2} \mathrm{~A}$ expressão "cuidador" faz referência às pessoas que prestam cuidados de prevenção, proteção e recuperação da saúde de maneira formal ou informal. O cuidador formal possui formação específica e, em geral, recebe remuneração pelo seu trabalho. Já o cuidador informal é leigo e, geralmente, é um membro da família que assume essa responsabilidade 3 .

A sobrecarga familiar é uma construção complexa que inclui diferentes domínios que vão desde as rotinas familiares relativas ao total de horas destinadas aos cuidados até as despesas extras no orçamento e redes sociais de apoio. Desta forma, entende-se que a compreensão da sobrecarga familiar deve ser realizada em âmbito biopsicossocial ${ }^{4}$. Assim, ao se analisar a sobrecarga da família, deve-se considerar o esforço físico em si despendido com o sujeito cuidado, o estresse gerado por este ato e o quanto das relações e atividades sociais da família são modificadas por conta da necessidade do ato de cuidar.

A sobrecarga cotidiana do cuidador é demonstrada pela estafante e estressora atividade de cuidados diários e ininterruptos. Por conta de tais cuidados, muitas vezes o cuidador necessita mudar sua rotina de atividades diárias, passando a conviver com a carga de trabalho dispensada ao cuidado da pessoa, que varia de acordo com a gravidade da condição de saúde ou deficiência ${ }^{5}$. Quanto maior a sobrecarga, menor a qualidade de vida do cuidador ${ }^{6}$.

Estudos têm tentado mensurar a sobrecarga e alguns aspectos da vida dos cuidadores e familiares mais afetados pela presença da pessoa com deficiência, além de examinarem as características dos cuidadores ${ }^{7,8}$. A Burden Interview é um dos instrumentos mais frequentemente utilizados para avaliar a sobrecarga de cuidadores ${ }^{7}$. Trata-se de um questionário originalmente elaborado para a avaliação de cuidadores de pessoas com esquizofrenia e, posteriormente, adaptado para cuidadores de pessoas com $\mathrm{DI}^{9}$. Este instrumento também tem sido atualmente utilizado para a avaliação nos casos de síndrome de Down (SD) ${ }^{6}$.

A SD ou trissomia do 21 é uma condição genética caracterizada pela presença de um cromossomo extra ou parte de um cromossomo extra, causando ao invés da duplicação, a triplicação do material genético referente ao par cromossômico $21^{10}$. O processo de aprendizagem das pessoas com SD é complexo e, em geral, exige um grau elevado adicional de suporte familiar e social ${ }^{11}$.

A família e os cuidadores enfrentam muitas dificuldades na tentativa da inclusão da pessoa com SD na sociedade. Entretanto essa dinâmica é um fator importantíssimo para a qualidade de vida e bem estar familiar ${ }^{8}$. As pessoas com SD contam com a família como o seu primeiro universo de relações sociais, tornando-se, em geral, permanentemente dependentes deste vínculo. Desta maneira, os seus familiares, além de serem seus responsáveis legais, assumem também a função de cuidadores ${ }^{3}$.

No Brasil, ainda existe pouca informação sistematizada sobre parentes e outros cuidadores informais de pessoas com deficiência ${ }^{12}$. Por outro lado, exis- 
te uma prevalência de uma pessoa com SD para cada 700 nascidos vivos ${ }^{13}$, além de se verificar um aumento da longevidade destes indivíduos. Fonseca et al. ${ }^{14}$ justificam a importância de investigações que contribuam para a elucidação da sobrecarga gerada aos seus cuidadores, uma vez que estes podem ter sua saúde comprometida pelo ato de cuidar.

Um dos fatores que poderia contribuir para a atenuação da sobrecarga dos cuidadores seria a melhora na autonomia dos indivíduos com $\mathrm{SD}^{15}$. No entanto, apesar de alguns autores sugerirem que a prática de exercícios físicos apresenta resultados favoráveis para a aptidão física e autonomia de indivíduos com $\mathrm{SD}^{16}$, o sedentarismo ainda é comum nessa população ${ }^{17}$. Algumas barreiras apontadas para justificar a elevada prevalência de sedentarismo seriam a falta de programas específicos, de profissionais capacitados e restrições impostas pela própria deficiência ${ }^{16,17}$.

Assim, tendo em vista a relevância do tema, o objetivo do presente estudo foi avaliar a sobrecarga de cuidadores de jovens com Síndrome de Down e a possível influência de dois programas de exercícios físicos sobre esta variável.

\section{MÉTODO}

\section{Participantes}

Participaram do estudo 38 adolescentes com SD com idades entre 12 e 20 anos de ambos os sexos. Participaram também os 38 cuidadores responsáveis pelos adolescentes, com idades entre 32 e 63 anos. Em todos os casos, os cuidadores avaliados foram as mães dos adolescentes. Todos os adolescentes participantes eram provenientes de instituições que prestavam assistência a pessoas com SD na cidade de Londrina - PR. Foram excluídos do estudo os adolescentes que apresentaram comprometimentos ortopédicos ou cardíacos, instabilidade atlanto-axial, que faziam uso de medicamentos que alteravam a frequência cardíaca e que tinham deficiência intelectual severa ou profunda, que impedisse a compreensão e/ou realização dos procedimentos.

Os 38 adolescentes foram divididos em três grupos, sendo GC - grupo controle- com 9 participantes; GTA - grupo de treinamento aeróbio - com 15 participantes; e GTR - grupo de treinamento resistido - com 14 participantes. A distribuição dos grupos foi realizada por conveniência, de acordo com a disponibilidade de comparecimento às atividades do programa. Os indivíduos que fizeram parte do grupo controle afirmaram não praticar atividade física de maneira regular e foram orientados a continuar suas atividades usuais normalmente. Para a participação no estudo, as mães, responsáveis legais pelos adolescentes, assinaram um Termo de Consentimento Livre e Esclarecido (TCLE). Este estudo obteve aprovação do Comitê de Ética em Pesquisa com Seres Humanos da Universidade Estadual de Londrina em consonância com o disposto na resolução nº 196/96 (Parecer 93.680/2012).

\section{Instrumentos}

\section{- Questionários}

Foram aplicados individualmente pelo pesquisador três questionários apenas aos cuidadores responsáveis pelos indivíduos com SD. O primeiro foi um questionário elaborado pela própria pesquisadora, composto por questões re- 
lacionadas à idade materna, escolaridade, idade, sexo do cuidador principal, dados sobre a prática de atividade física habitual e condições de saúde associadas dos adolescentes com SD.

O segundo questionário foi sobre o nível socioeconômico das famílias, elaborado pela Associação Brasileira de Empresas de Pesquisa ${ }^{18}$. Neste questionário é considerada a posse de bens e o grau de instrução do chefe da família para classificar os indivíduos nas classes econômicas: A1, A2, B1, B2, C1, C2, $\mathrm{D}$ e E. No presente estudo, para melhor visualização dos resultados, as classes A1 e A2 foram agrupadas em A, as classes B1 e B2 foram agrupadas em B e as classes $\mathrm{C} 1$ e $\mathrm{C} 2$ foram agrupadas em $\mathrm{C}$.

O terceiro questionário aplicado foi o Burden Interview (Anexo 1) para avaliação da percepção de sobrecarga do cuidador, validado para cuidadores de indivíduos com deficiência intelectual ${ }^{9}$ em sua versão adaptada para a língua portuguesa $^{7}$. Esse questionário é composto por 22 itens que avaliam a relação do cuidador com a pessoa cuidada, sua condição de saúde, bem estar psicológico, finanças e vida social. As 21 primeiras questões avaliam objetiva e subjetivamente a sobrecarga e as respostas são pontuadas por uma escala Likert de acordo com a intensidade da resposta para cada afirmativa $(0=$ nunca, $1=$ raramente, 2 =algumas vezes, $3=$ frequentemente e $4=$ =sempre). A última questão é geral e busca avaliar o quanto o cuidador considera estar sobrecarregado ao desempenhar a sua função. Neste item, as respostas possíveis são: $0=$ nem um pouco; 1 = um pouco; 2 = moderadamente; 3 = muito 4 = extremamente. A pontuação total varia de 0 a 88 e, quanto maior a percepção de sobrecarga, maiores as pontuações ${ }^{19,20}$. A Tabela 1 a seguir mostra a forma de classificação da sobrecarga dos cuidadores, de acordo com a pontuação total obtida na Burden Interview.

TABELA 1 - Classificação do Nível de Sobrecarga de Cuidadores19,20

\begin{tabular}{lc}
\hline Escore & Nível de Sobrecarga \\
\hline$>21$ & Ausência ou pouca sobrecarga \\
\hline 21 e 40 & Sobrecarga moderada \\
\hline 41 e 60 & Sobrecarga moderada a severa \\
\hline 60 a 88 & Sobrecarga Severa \\
\hline
\end{tabular}

\section{- Programas de treinamento}

Os adolescentes dos grupos de treinamento aeróbio(GTA) e treinamento resistido (GTR) foram submetidos a programas de treinamento durante 12 semanas, com duração de 50 minutos por sessão. Os treinamentos foram realizados na academia do Centro de Educação Física e Esporte, da Universidade Estadual de Londrina.

O treinamento aeróbio foi realizado três vezes por semana, com duração de 30 minutos, sendo 15 minutos em esteira e 15 minutos em bicicleta ergométrica, com intensidade entre 50 e $70 \%$ da FCreserva, precedido de $10 \mathrm{mi}-$ nutos de aquecimento (articular e alongamento) e sucedido de mais 10 minutos de recuperação (alongamento). Para monitorar a intensidade foi utilizado o monitor de frequência cardíaca da marca Polar FT2.

Já para o GTR foi proposto um programa de treinamento resistido duas vezes por semana com nove exercícios em três séries de 12 repetições para cada, com intervalo de um minuto entre as séries e de três minutos entre os 
exercícios. A seguinte série de exercícios foi proposta: supino máquina, cadeira extensora, puxada aberta frontal, bíceps cabo, flexora em pé com caneleira, tríceps cabo, panturrilha com caneleira, elevação frontal com halter e abdominais. Foram realizadas duas sessões iniciais com cargas leves para adaptação ao exercício e, depois disso, a carga utilizada foi estimada observando-se a capacidade de realização do exercício em 12 repetições. A progressão da carga foi espontânea, sendo aumentada à medida que o indivíduo conseguisse realizar as três séries com 12 repetições completas ${ }^{21}$.

\section{Análise Estatística}

Os dados foram inicialmente tratados por meio da estatística descritiva, apresentada por valores mínimos e máximos, média e desvio-padrão para as variáveis contínuas e frequência de respostas para as variáveis categóricas. Para comparação entre os resultados pré e pós treinamento do Burden Interview, foi utilizado o Teste t-student pareado. Para a comparação dos resultados do Burden Interview entre as diversas classes socioeconômicas foi utilizada ANOVAone -way com post hoc de Tukey. Em todas as situações foi adotada significância p $\leq$ 0,05. Os dados foram tabulados e analisados no programa estatístico SPSS 18.0.

\section{RESULTADOS}

Os 38 adolescentes participantes apresentaram idade média de 15,7 anos ( \pm $2,7)$, sendo a maior parte do sexo masculino (63,2\%).Deste total, $34,2 \%$ apresentava hipotireoidismo (Tabela 2). Já entre os 38 cuidadores, todas eram mães dos jovens com SD. A idade média destes cuidadores era de 49,8 anos $( \pm 8,3)$. Com relação ao nível socioeconômico, $5,3 \%$ das famílias pertencia à classe $\mathrm{D}$, $55,3 \%$ à classe $\mathrm{C}, 39,5 \%$ à classe $\mathrm{B}$ e nenhum deles às classes $\mathrm{A}$ ou $\mathrm{E}$ (Tabela 3 ).

TABELA 2 - Caracterização dos participantes - Jovens com SD

\begin{tabular}{lc}
\hline Sexo & $\%(n)$ \\
\hline Masculino & $63,2(24)$ \\
Feminino & $36,8(14)$ \\
\hline Hipotireoidismo & $\%(n)$ \\
\hline Sim & $34,2(13)$ \\
Não & $65,8(25)$ \\
\hline
\end{tabular}

TABELA 3 - Caracterização dos participantes - Cuidadores

\begin{tabular}{ll}
\hline Escolaridade & $\%(\mathrm{n})$ \\
\hline Fundamental Incompleto & $36,8(14)$ \\
Fundamental Completo & $18,4(7)$ \\
Médio Incompleto & $7,9(3)$ \\
Médio Completo & $31,6(12)$ \\
Superior Incompleto & $0(0)$ \\
Superior Completo & $5,3(2)$ \\
Pós Graduação & $0(0)$ \\
\hline Nível Socioeconômico & $\%(n)$ \\
\hline A & $0(0)$ \\
B & $39,5(15)$ \\
C & $55,2(21)$ \\
D & $5,3(2)$ \\
E & $0(0)$ \\
\hline
\end{tabular}


Em relação aos resultados do Burden Interview pré e pós treinamento, não foi observada diferença significante (Tabela 4), assim como também não houve diferença entre os tipos de exercício(aeróbio $\mathrm{p}=0,351$ e resistido $\mathrm{p}=0,605$ ) e o grupo controle $(\mathrm{p}=0,608)$. Por fim, também não foi encontrada diferença entre os cuidadores dos participantes com e sem diagnóstico dehipotireoidis$\operatorname{mo}(\mathrm{p}=0,451)$.

TABELA 4 - Resultados das pontuações obtidas no Burden Interview pré e pós-intervenção

\begin{tabular}{lccc}
\hline & $\begin{array}{c}\text { Pontuação } \\
\text { Mínima }\end{array}$ & $\begin{array}{c}\text { Pontuação } \\
\text { Máxima }\end{array}$ & Média $( \pm$ Desvio padrão) \\
\hline Burden Interview Pré-intervenção & 4 & 69 & $25,13( \pm 13,0)$ \\
\hline Burden Interview Pós-intervenção & 6 & 72 & $27,13( \pm 15,2)$ \\
\hline
\end{tabular}

De acordo com os resultados da análise de variância, foi observada uma sobrecarga maior dos cuidadores com nível sócio econômico caracterizado como classe $\mathrm{D}$, quando comparada às classes $\mathrm{C}$ e $\mathrm{B}(\mathrm{p}=0,008)$ e na classe $\mathrm{C}$ quando comparada à classe $\mathrm{B}(\mathrm{p}=0,026)$. A escolaridade e a idade do cuidador não interferiram na percepção desobrecarga.

\section{DISCUSSÃO}

A maior parte dos estudos que avaliaram a percepção de sobrecarga de cuidadores concentra-se em cuidadores de idosos, de pessoas com doenças demenciais e com outras doenças crônicas ${ }^{6,22,23}$. Entretanto, as características dos cuidadores em relação ao gênero e média de idade aqui encontradas são semelhantes às observadas nestes estudos e também em estudos com cuidadores de pessoas com deficiência intelectual ${ }^{6,12,24,25}$. Tais estudos apontam que, normalmente,a posição de cuidar da família é assumida pelas mães, enquanto o homem cuida do trabalho externo. Este fato também foi evidenciado no presente estudo, uma vez que todos os cuidadores pesquisados foram as mães dos adolescentes.

Apesar dos escores totais do Burden Interviewpré e pós-intervenção apontarem para uma "sobrecarga moderada", verificou-se que, ao responderem à última questão sobre qual a sobrecarga sentida, 68,3\% apontaram não sentir "nem um pouco" ou apenas "um pouco" a sobrecarga no momento pré-intervenção, contra 56,1\% dos cuidadores no momento pós-intervenção. Estes dados mostram que, aparentemente, esses cuidadores apresentam dificuldade em admitir de maneira direta a sobrecarga sentida.

A dificuldade do cuidador em assumir diretamente a sobrecarga também foi observada em outros estudos ${ }^{6,12,23}$. Ainda segundo Santos et al. ${ }^{24}$, não se pode descartar a possibilidade do escore relativo à sobrecarga sofrer interferência pelo fato de alguns entrevistados ficarem com algum receio ou até mesmo com vergonha de responder honestamente sobre sua relação com a tarefa de cuidar.Por outro lado, uma percepção subestimada da sobrecarga pode ser atribuída a questões como o vínculo afetivo mãe-filho e à impossibilidade de delegar essa função, já assumida como projeto de vida ${ }^{24}$. Os genitores vivenciam diversos sentimentos em relação ao seu filho com deficiência, tais como raiva, rejeição, revolta e culpa, apresentando as mães mais tendência a estes 
sentimentos do que os pais ${ }^{3}$. Talvez este seja mais um motivo para que as mães cuidadoras tenham dificuldade em admitir a real sobrecarga sentida.

A "sobrecarga moderada" encontrada pelos escores totais no presente estudo corroboram os resultados encontrados por Camargos et al..$^{22}$, que ao investigar a sobrecarga em cuidadores de crianças com paralisia cerebral, obtiveram uma média de 21,95. Da mesma forma, Interdonato et al. ${ }^{26}$, analisando a sobrecarga de cuidadores de pessoas com deficiência física e intelectual, obtiveram um escore médio de 22,6, também sendo enquadrado como "sobrecarga moderada". Amaral et al. ${ }^{6}$, também em estudo com cuidadores de pessoas com síndrome de Down, observaram 53\% dos cuidadores com "sobrecarga moderada" e apenas 10\% com "sobrecarga moderada a severa".

No estudo de Irazal et al. ${ }^{4}$ foram encontrados baixos níveis de sobrecarga familiar de pessoas com DI. Segundo os autores, a amostra foi constituída por famílias hispânicas, que demonstrariammaior aceitação da deficiência. Neste mesmo estudo, valores mais altos de sobrecarga foram relatados em situações nas quais, além da deficiência intelectual, estava presente também alguma desordem mental. As variáveis clínicase funcionais da deficiência,que explicaramgrausmais elevados de sobrecargafamiliar no caso de pessoas com DI com desordens mentais, foram a presença dedistúrbios comportamentais, afetivosepsicóticose maioresdeficiênciasnas áreas departicipação na sociedade e cuidados pessoais.

As áreas relacionadas à incapacidade funcional, à dificuldade de participação social e aos cuidados pessoais são as que mais contribuem para uma carga familiar maior. Alguns autoresindicamque a deficiênciaem contextos sociaispode explicarasobrecarga familiar ${ }^{27}$. A menor sobrecarga familiar percebida nas situações de DI sem desordens mentais também foi encontrada por Martorell et $\mathrm{al}^{1}$. Neste mesmo estudo foram relatadas diferenças significantesrelacionadas à idade do cuidador, evidenciando que omaior nível de sobrecarga estava associado à idade mais jovem (<27 anos). A menor sobrecarga relacionada ao aumento da idade pode ser entendida como resultado de um processo de adaptação.No presente estudo, a média de idade dos cuidadores foi de 49,8 anos, o que pode justificar, em parte, o não aparecimento da percepção de "sobrecarga severa" na amostra.

Em estudos que avaliaram a sobrecarga de cuidadores de idosos, os autores relataram maiores escores de sobrecarga. Oliveira et al..$^{25}$ e Gratão et al. ${ }^{28}$ encontraram escores médios de 31,9 e 27,8, respectivamente. Essa diferença pode ocorrer porque, provavelmente, é mais fácil assumir a sobrecarga nos cuidados com um idoso do que com uma criança ou jovem ${ }^{29}$.

Outro dado importante verificado no presente estudo foi a sobrecarga maior nos cuidadores com menor nível socioeconômico. Esse resultado corrobora achados de outros estudos que apontam que a desvantagem socioeconômica e cultural pode interferir nos níveis de estresse dos pais/cuidadores e está relacionada a um pior relacionamento entre pais e filhos ${ }^{26}$. Camargos et al. ${ }^{23}$ também reportaram em seu estudo maior sobrecarga dos cuidadores em famílias com condições socioeconômicas menos favoráveis. Além disso, os autoresafirmam que os cuidadores de crianças com deficiência com nível socioeconômico elevado controlam melhor o seu bem estar quando comparados com cuidadores com nível socioeconômico inferior.

Em seu estudo, Gallagher et al..$^{30}$ (2012) destacaramasligações entreo apoio social, estresse e saúde física, e afirmaram queos pais de criançascom defi- 
ciência intelectualcommaior apoio socialreportarammenos estresse emelhor saúde físicaem comparação com aquelescomrelações sociaismais pobres.Por outro lado, alguns autores não encontraram relação entre a percepção de sobrecarga do cuidadore a renda familiar ${ }^{6,24}$.

Neste estudo, não foi observada influência do programa de exercício físico na sobrecarga dos cuidadores. Possivelmente, o pouco tempo de treinamento e a importância da variável socioeconômica pode ter dificultado a observação de diferença da sobrecarga pré e pós-intervenção. Além disso, como a sobrecarga inicial foi considerada moderada, fica mais difícil a observação de uma redução significante.

Apesar disso, cabe destacar que as atividades corporais melhoram a autonomia e o autocuidado das pessoas com $\mathrm{SD}^{10}$. Além disso, pessoas com esta condição tendem a apresentar menores índices de atividade física e, por esse motivo, estão mais suscetíveis às doenças secundárias e crônicas provenientes do estilo de vida sedentário 9 . Por esses motivos, ressalta-se que a prática regular de exercícios físicos é muito importante para a qualidade de vida desses jovens.

Entende-se, ainda, como limitações do presente estudo a amostra reduzida e o fato de ser selecionada por conveniência. Entretanto, estudos com pessoas com deficiência dificultam a seleção aleatória de amostra, sobretudo pela dificuldade em se localizar os participantes fora das instituições de assistência específicas. Por fim, o pouco tempo de intervenção, como citado anteriormente, também pode ser considerado um fator limitante do estudo. Apesar disso, acredita-se que essa pesquisa contribui para a compreensão dos efeitos de sobrecarga a que são submetidos os cuidadores de jovens com SD e para a reflexão de formas de intervenção para minimizar possíveis prejuízos à qualidade de vida desses cuidadores.

\section{CONCLUSÃO}

O presente estudo apresentou dados referentes à sobrecarga de cuidadores de jovens com SD. Os cuidadores desses jovens apresentaram dificuldade em relatar a sobrecarga que os cuidados prestados acarretam para eles, o que talvez possa ser explicado pelo laço familiar maternal existente entre eles.

A realização de um programa de treinamento físico não se mostrou suficiente para influenciar a percepção da sobrecarga dos cuidadores, não sendo observadas diferenças entre os jovens submetidos à intervenção e aqueles do grupo controle. No entanto, pode-se concluir que existe uma tendência ao aumento da sobrecarga para cuidadores de nível socioeconômico mais baixo.

Os cuidadores de pessoas com deficiência apresentam, em longo prazo, consequências na saúde física, emocional e funcional. A atenção prestada às pessoas responsáveis pelos cuidados destas crianças e jovens só será realizada de forma adequada quando houver conhecimento profundo e sistematizado sobre o assunto. Por esse motivo, programas com objetivo de promover a saúde do cuidador deveriam ser inseridos no processo de reabilitação da criança e do jovem com deficiência. Desta forma, os cuidados prestados à criança, provavelmente, seriam melhorados, e possíveis desgastes do cuidador seriam minimizados. 


\section{REFERÊNCIAS}

1. Martorell A, Gutierrez-Recacha P, Irazábal M, Marsa F, Garcia M. Family impact in intellectual disability, severe mental health disorders and mental health disorders in ID. A comparison. ResDev Disabil.2011;32:2847-52.

2. Blacher J, Baker BL, Mclean WE. Positive impact of intellectual disability on families. Am J Ment Retard.2007; 112(5):330-48.

3. Silva CF, Passos VM, Barreto SA. Frequência e repercussão da sobrecarga de cuidadoras familiares de idosos com demência. RevBrasGeriatrGerontol. 2012; 15(4):707-31.

4. Irazábal M, Marsa F, Garcia M, Gutierrez-Recacha P, Martorell A, Salvador-Carulla L, et al.Family burden related to clinical and functional variables of people with intellectual disability with and without a mental disorder. ResDev Disabil.2012; 33:796-803.

5. Ribeiro AF, Souza C. O cuidador familiar de doentes com câncer. Arquivos de Ciências da Saúde.2010; 17(1):22-7.

6. Amaral EG, Mendonça MS, Prudente COM, Ribeiro MFM. Qualidade de vida e sobrecarga em cuidadores de crianças com síndrome de Down. Rev Movimenta. 2011; 4 (2): 99-108.

7. Scazufca M. Brazilian version of the Burden Interview scale for the assessment of Burden of care in careers of people with mental illnesses. Rev Bras Psiquiatr.2002;24 (1):12-7

8. Murphy NA, Christian B,Caplin DA, Young PC. The health of caregivers for children with disabilities: caregiver perspectives. Journal Compilation Child: care, health and development. 2006;33 (2):180-7.

9. Martorell A, Pereda A, Salvador-Carulla L, Ochoa S, Yuso-Mateos JL. Validation of the subjective and objective Family Burden Interview (SOFBI/ECFOS) in primary caregivers to adult with intellectual disabilities living in community. J IntellectDisabil Res. 2007; 51 (2):892-901.

10. Martins MRI, Fecuri MAB, Arroyo MA, Parisi MT. Avaliação das habilidades funcionais e de auto cuidado de indivíduos com síndrome de Down pertencentes a uma oficina terapêutica. Rev CEFAC.2013;15(2): 361-5.

11. Pazin AC, Martins MRI. Desempenho funcional de criança com síndrome de Down e a qualidade de vida de seus cuidadores. Rev Neurocienc.2007;15 (4):297-303.

12. Pimenta RA, Rodrigues LA, Greguol M. Avaliação da qualidade de vida e sobrecarga de cuidadores de pessoas com deficiência intelectual. RevBrasci Saúde. 2010;14(3): 69-76.

13. Conselho Nacional de Saúde [internet]. Prevalência de nascidos vivos com Síndrome de Down [atualizado em 2012 maio; citado 2012 maio]. Disponível em: url://www. conselho.saude.gov.br .

14. Fonseca LM, Gonçalves AS, Stocco L, Carvalho CL, Matos MT, Silva HCS, et al. Implicações sobre a deficiência intelectual e longevidade: Projeto piloto de intervenção em casos de senilidade. FedNac das Apaes. 2013; 1(2):3-21.

15. Zani AV, Merino MFGL, Marcon SS. The feelings and experience of a mother facing Down syndrome. Acta Scientiarum. 2013;35 (1):67-75.

16. Modesto E, Greguol M. Influência do treinamento resistido em pessoas com síndrome de Down - uma revisão sistemática. Rev Bras AtivFis Saúde.2014; 19 (2):153-67.

17. Hinckson EA, Curtis A. Measuring physical activity in children and youth living with intellectual disabilities: a systematic review. Res DevDisabil. 2013; 34:72-86

18. Associação Brasileira das Empresas de Pesquisa [internet] Critério de classificação econômica Brasil. [atualizado em 2012 maio; citado 2012 junho] Disponível em url:// www.abep.org.br.

19. Kron CA, Ballarin MLGS. Avaliação da sobrecarga em cuidadores: um estudo derevisão sobre a escala ZaritBurden interview. Anais do XVII Encontro de Iniciação Científica PUC Campinas. 2013;43-53.

20. Wachholz PA, Santos RCC, Wolf LSP. Reconhecendo a sobrecarga e a qualidade de vida em cuidadores familiares de idosos frágeis. Rev Bras GeriatGerontol. 2013;16 (3):513-526.

21. American College of Sports Medicine. ACMS's Guidelines for Exercise Testing and Prescription, Eighth Edition, Baltimore: 2009. 
22. Camargos ACR, Lacerda TTB, Viana SO, Pinto LRA, Fonseca MLS. Avaliação da sobrecarga do cuidador da criança com paralisia cerebral através da escala Burden Interview. Rev BraSaúde Mater Infant. 2009; 9 (1): 31-7.

23. Ruiz-Robledillo N, Garcia SA, Blasco JP, Bono EG, Albiol LM. Highly resilient coping entails better perceived health, high social support and low morning cortisol levels in parents of children with autism spectrum disorder. Res Dev Disabil.2014; 35:686-95.

24. Santos AAS, Vargas MM, Oliveira CCC, Macedo IAB. Avaliação da sobrecarga dos cuidadores de crianças com paralisia cerebral. CiencCuid Saúde. 2010; 9(3): 503-9.

25. Oliveira DC, Carvalho GSF, Stella F, Higa CMH, Delboux MJ. Qualidade de vida e sobrecarga de trabalho em cuidadores de idosos em seguimento ambulatorial. Texto Contexto Enferm.2011; 20 (2): 234-40.

26. Interdonato GC, Pinto IC, Takahara L, Moraes J, Bertola T, Pinto RR. Cuidando de quem cuida: avaliando a sobrecarga de cuidadores de crianças com deficiência. $\mathrm{V}$ Congresso multiprofissional em Saúde UniFil. 2014: 404-7.

27. Maes B, Broekman TG, Dosen A, Nauts J. Care giving Burden of families looking after persons with intellectual disability and behavioral or psychiatric problems. J Intellect Disabil Res. 2003; 47: 447-55.

28. Gratão ACM, Talmelli LFS, Figueiredo LC, Rosset I, Freitas CP, Rodrigues RAP. Dependência funcional de idosos e a sobrecarga do cuidador. RevEscEnferm USP.2013; 47 (1):137-44.

29. Rosset MOS. A sobrecarga na família de crianças com paralisia cerebral [dissertação de mestrado]. Ribeirão Preto: Universidade de São Paulo; 2009.

30. Gallagher S, Whiteley J. The association between stress and physical health in parents caring for children with intellectual disabilities is moderated by children's challenging behaviors. JHealth Psychol. 2012; 18 (9): 1220- 31.

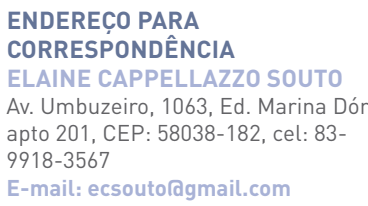

\section{ANEXO 1}

\section{Burden Interview (SCAZUFCA, 2002)}

INSTRUÇÕES: A seguir encontra-se uma lista de afirmativas que refletem como as pessoas algumas vezes sentem-se quando cuidam de outra pessoa. Depois de cada afirmativa, indique com que frequência o $\mathrm{Sr} / \mathrm{Sra}$ se sente daquela maneira(nunca $=0$, raramente $=1$, algumas vezes $=2$, frequentemente $=3$, ou sempre=4). Não existem respostas certas ou erradas.

1. O Sr/Sra sente que $\mathrm{S}^{*}$ pede mais ajuda do que ele (ela) necessita?

2. O Sr/Sra sente que por causa do tempo que o $\mathrm{Sr} / \mathrm{Sra}$ gasta com $\mathrm{S}$, o Sr/Sra não tem tempo suficiente para si mesmo (a)?

3. O Sr/Sra se sente estressado (a) entre cuidar de $\mathrm{S}$ e suas outras responsabilidades com a família e o trabalho?

4. O Sr/Sra se sente envergonhado (a) com o comportamento de S?

5. O Sr/Sra se sente irritado (a) quando S está por perto?

6. O Sr/Sra sente que $\mathrm{S}$ afeta negativamente seus relacionamentos com outros membros da famìlia ou amigos? 
7. O Sr/Sra sente receio pelo futuro de $\mathrm{S}$ ?

8. O Sr/Sra sente que $\mathrm{S}$ depende do $\mathrm{Sr} / \mathrm{Sra}$ ?

9. O Sr/Sra se sente tenso (a) quando $\mathrm{S}$ esta por perto?

10. O Sr/Sra sente que a sua saúde foi afetada por causa do seu envolvimento com $\mathrm{S}$ ?

11. O Sr/Sra sente que o Sr/Sra não tem tanta privacidade como gostaria, por causa de $S$ ?

12. O Sr/Sra sente que a sua vida social tem sido prejudicada porque o $\mathrm{Sr} / \mathrm{Sra}$ está cuidando de $\mathrm{S}$ ?

13. $\mathrm{O} \mathrm{Sr/Sra}$ não se sente à vontade de ter visitas em casa, por causa de $\mathrm{S}$ ?

14. O Sr/Sra sente que $\mathrm{S}$ espera que o $\mathrm{Sr} / \mathrm{Sra}$ cuide dele/dela, como se o $\mathrm{Sr} / \mathrm{Sra}$ fosse a única pessoa de quem ele/ela pode depender?

15. O Sr/Sra sente que não tem dinheiro suficiente para cuidar de $S$, somandose as suas outras despesas?

16. O Sr/Sra sente que ser incapaz de cuidar de $\mathrm{S}$ por muito mais tempo?

17. $\mathrm{O} \mathrm{Sr} / \mathrm{Sra}$ sente que perdeu o controle da sua vida desde a doença de $\mathrm{S}$ ?

18. O Sr/Sra gostaria de simplesmente deixar que outra pessoa cuidasse de $\mathrm{S}$ ?

19. O Sr/Sra se sente em dívida sobre o que fazer por S?

20. $\mathrm{O} \mathrm{Sr} / \mathrm{Sra}$ sente que deveria estar fazendo mais por $\mathrm{S}$ ?

21. O Sr/Sra sente que poderia cuidar melhor de $\mathrm{S}$ ?

22.De uma maneira geral, quanto o Sr/Sra se sente sobrecarregado (a) por cuidar de $\mathrm{S}^{* *}$ ?

*No texto $S$ refere-se a quem é cuidado pelo entrevistado.

**Neste item as respostas são: nem um pouco $=0$, um pouco $=1$, moderadamente $=2$, muito $=3$, extremamente $=4$. 